\title{
Variations
}

Variations

Revue internationale de théorie critique

$16 \mid 2012$

Tahrir is here !

\section{La résistance, un principe sociologique à l'œuvre}

\section{Alexander Neumann}

\section{OpenEdition}

Journals

Édition électronique

URL : http://journals.openedition.org/variations/141

DOI : 10.4000/variations. 141

ISSN : 1968-3960

\section{Éditeur}

Les amis de Variations

\section{Édition imprimée}

Date de publication : 16 janvier 2012

\section{Référence électronique}

Alexander Neumann, «La résistance, un principe sociologique à l'œuvre », Variations [En ligne],

16 | 2012, mis en ligne le 20 février 2012, consulté le 19 avril 2019. URL : http://

journals.openedition.org/variations/141; DOI : 10.4000/variations. 141

Ce document a été généré automatiquement le 19 avril 2019.

Les ami•e•s de Variations 


\title{
La résistance, un principe sociologique à l'œuvre
}

\author{
Alexander Neumann
}

\section{NOTE DE L'ÉDITEUR}

Première publication sur www.theoriecritique.com, « Tahrir is here ! ", 16 janvier 2012, pp. 70-78

1 La résistance a subjugué libraires, lectrices et lecteurs grâce au court ouvrage de Stéphane Hessel, lui-même issu de la résistance au nazisme ${ }^{1}$. Ce best-seller des bestsellers du nouveau siècle s'organise autour du leitmotiv Résister c'est créer, créer c'est résister. En sciences sociales en revanche, le concept de résistance n'a pas la cote de nos jours. C'est probablement la raison pour laquelle il s'est enfui des universités pour parcourir les rues des quatre coins du monde, afin de se muer en mot d'ordre contemporain dans toutes les langues, de Tunis à New York et de Tel Aviv à Santiago de Chile en passant par Athènes et Madrid.

2 Au sein de la sociologie européenne contemporaine en effet, le concept de résistance est généralement ignoré, lorsqu'il n'est pas associé à un phénomène pré-moderne, à une obstination réactionnaire ou à un avatar sans avenir du marxisme doctrinaire. En un mot, ce serait ringard. Dans un essai sur la théorie sociologique publié en 1968, on trouve déjà épinglée la tendance professorale bien répandue à rationaliser tous les problèmes sociaux et à rechercher systématiquement les voies de l'adaptation individuelle et culturelle, par exemple chez Touraine et Crozier ${ }^{2}$. L'auteur de cet essai, un certain Cohn-Bendit, poursuit son argumentation en situant le principal potentiel critique du côté de la résistance qu'exerce alors le mouvement étudiant.

3 La résistance pourrait donc passer pour un concept autant extra-parlementaire qu'extrauniversitaire, s'il ne se trouvait des sociologues et philosophes pour le sauver de cette barbarie postmoderne où il fait bon vivre et penser comme des porcs ${ }^{3}$. La même année, dans son adresse inaugurale, le président du congrès de sociologie allemande nommait en 
effet les principes qui pourraient soutenir une conceptualisation critique des sociétés occidentales, menacées d'une nouvelle régression systémique :

«La résistance à l'encontre de l'adaptation aveugle, la liberté de choisir ses propres objectifs en toute raison, la nausée face à un monde fait de mensonge et de spectacle, la compréhension des possibilités du changement. $»^{4}$

Tels sont les principes qui devraient ouvrir l'horizon d'une sociologie critique, selon Theodor W. Adorno en 1968 - une sociologie qui ne devrait pas se borner à produire des informations bienvenues au profit des agences et des intérêts établis, précise-t-il.

La situation de crise globale ouverte en 2008 - comparée à la crise mondiale de 1929 par des observateurs aussi avertis que Paul Krugman - rend à mon sens légitime la relance de concepts sociologiques originaux qui semblaient éloignés des centres d'intérêt de la sociologie européenne: la résistance, la subjectivité rebelle, l'espace public oppositionnel. Appliqué aux phénomènes européens, le décentrement critique que je propose pourrait se présenter, in fine, comme un recentrement de la sociologie sur sa propre tradition critique, qui fut marquée par la crise globale de 1929, par la défaite du fascisme européen et 1968. Avec l'effondrement de l'autorégulation des marchés financiers depuis 2008, la crise de régime de plusieurs Etats européens, la théorie des systèmes a manifestement rencontré sa limite. Sur un plan théorique, ce constat interroge aussi l'importation de ces approches dans l'agir communicationnel d'Habermas ${ }^{5}$. Aujourd'hui, l'érosion des normes collectives, les problèmes de légitimation du capitalisme et la distanciation massive à l'égard de la représentativité démocratique de l'Etat favorisent une expressivité politique fondée sur l'expérience sensible des acteurs. Pareille expérience fondamentale engendre une prise de parole qui se déploie dès qu'un espace susceptible de l'accueillir apparaît. La crise globale produit de tels espaces avec une grande probabilité6. En 2011, le surgissement d'espaces publics oppositionnels de toutes sortes se produit à un tel rythme que la sociologie politique peine à en saisir toute l'étendue, des révolutions maghrébines et de la contestation israélienne aux mouvements européens - résumés par le leitmotiv Indignados - en passant par la Constituante islandaise, jusqu'à la crise politique en Inde, provoquée par un vaste mouvement de désobéissance et au mouvement Occupy Wall Street qui s'étend à travers l'Amérique du Nord. Il s'agit par conséquent de saisir les phénomènes de débordement de la normativité que la sociologie positiviste n'est pas en mesure de nommer. L'érosion de normes, les pratiques transgressives ou contestataires, les effets politiques des crises sociales à l'œuvre, ne sauraient être cernés à travers des enquêtes de terrain limitées ou des statistiques qui cherchent à reproduire les critères factuels de la gestion institutionnelle. En d'autres termes, il semble prometteur de relancer une sociologie critique de la crise que j'associe à la Théorie critique et au courant chaud de l'Ecole de Francfort.

La Théorie critique, connue sous l'appellation d'Ecole de Francfort, n'est pas souvent associée à la sociologie, surtout dans l'espace francophone. La principale intervention sociologique d'Adorno, au congrès de sociologie allemande de 1968 qu'il présida, affirme que la sociologie doit avoir pour objet la critique du capitalisme, et non pas de la société industrielle, et s'élève explicitement contre la thèse selon laquelle la référence à Marx serait devenue désuète, ce qui implique en même temps une déconstruction de la vulgate marxiste d'influence stalinienne ${ }^{7}$. Des concepts clés de l'argumentation marxienne tels la plus-value, le fétichisme de la marchandise ou l'échange abstrait traversent la vie et l'œuvre d'Adorno tel un fil rouge. Cette interprétation de l'orientation d'Adorno que je 
défends pour ma part ${ }^{8}$, vient d'être systématiquement retracé depuis par Dirk Braunstein, dans un livre de 444 pages qu'il consacre à la Critique de l'économie politique d'Adorno9.

7 Sur le plan conceptuel, Adorno distingue nettement les concepts marxiens de la tradition marxiste, ce qui lui vaut d'être attaqué par les marxistes doctrinaires allemands ${ }^{10}$. Cela suppose de préciser la manière dont Karl Marx comprend le capital en tant qu'une totalité abstraite, à travers des concepts tels le fétichisme de la marchandise et la soumission réelle du salariat. Concepts qui se trouvent aussi soulignés par Adorno dans un article au sujet de "Marx et les catégories fondamentales de la sociologie $»^{11}$. Cette lecture contraste singulièrement avec la présentation que Honneth fait de la sociologie d'Adorno, ce que montre un petit exercice lexicométrique des deux principaux articles au sujet de la critique du capitalisme chez Adorno. Dans sa propre intervention au congrès de sociologie de 1968, Adorno ne se réfère pas moins de vingt-sept fois explicitement à Marx, et ne mentionne pas une seule fois Weber. Dans l'article qu'Honneth a publié en hommage à la critique adornienne du capitalisme en 2003, les références d'Adorno à Marx n'apparaissent pas une seule fois, notes comprises, tandis que la critique adornienne du capitalisme y est exclusivement interprété sur la base des thèses de Weber. Il est vrai que Honneth ne se voit pas comme " un élève d'Adorno » et préfère se "rapprocher du type de théorie de la société qui avait déjà été développée des classiques: Emile Durkheim, Max Weber et Talcot Parsons $»^{12}$. Pour sa part, Adorno a cherché à déconstruire les cadres théoriques de ces classiques, en faveur d'une sociologie de la crise capitaliste, comme le montrent son intervention de 1968 ou encore sa préface à la première traduction allemande de l'ouvrage d'Emile Durkheim, Sociologie et philosophie.

8 L'argumentation adornienne au sujet de Durkheim reste méconnue dans l'espace francophone. Adorno comprend le besoin initial de légitimer la sociologie en tant que discipline académique distincte, mais il souligne aussitôt la perte implicite ainsi que le non-dit que cela comporte. En traitant les « faits sociaux » comme des faits positifs, c'està-dire comme des choses, Durkheim réduit la base de la connaissance sociologique à l'observation des règles sociales manifestes, en dissociant radicalement la dimension individuelle-psychologique d'une part et l'objectivité sociale d'autre part, ce qui conduit à l'élimination de toute interprétation des motivations subjectives des membres de la société. Par conséquent, Durkheim n'arrive pas à discerner proprement les différents niveaux de représentation ou de "conscience » des acteurs sociaux, ni à distinguer les frontières entre des comportements "normaux » et "pathogènes ». Afin de conjurer ces difficultés épistémologiques, le sociologue français écarte les expériences vécues qui motivent les processus sociaux pour les remplacer par la représentation prétendument objective et unitaire de la "conscience collective». En procédant ainsi, Durkheim est obligé de désigner la conscience ou l'esprit de la société comme étant sa substance, ce qui signifie que la subjectivité revient dans la sociologie objective par la petite porte. Adorno conclut: "La vengeance du subjectivisme s'opère à travers les traits spirituels de l'objectivité, subjectivisme que nie Durkheim mais qui s'avère indispensable dans son approche. »

Dans la sociologie durkheimienne, le collectif social se présente comme une abstraction, qui veut regrouper les facultés de tous les individus qui composent l'ensemble social sous la forme d'une motivation générique, la "conscience collective ». Dans le même temps, Durkheim s'élève contre la désagrégation qui menace ce bel ensemble face aux conflits qui éclatent entre le travail et le capital, opinion normative qui culmine, en 1887, avec l'idée que les révolutions seraient aussi impossibles que des miracles. Il va de soi que la critique adornienne de Durkheim prépare le concept d'espace public oppositionnel, là où 
l'idée de conscience collective ne laisse de place ni au potentiel oppositionnel, ni à des comportements de résistance. L'histoire du 20ème siècle est emplie de miracles que Durkheim ne voulait pas imaginer, de cauchemars et de résistances.

Dans le même mouvement intellectuel, Adorno a développé une critique globale de la théorie de Weber. Il constate que celui-ci dissimule ses propres présupposés subjectifs derrière le postulat selon lequel les actions sociales seraient guidées par la rationalité des fins. Cette rationalité des actions sociales permet une compréhension sociologique rationnelle des acteurs et assure une certaine objectivité de la connaissance. Selon Adorno, cette évaluation des motivations des acteurs se fonde cependant sur une appréciation subjective du sociologue. Il constate que, chez Weber, « la position centrale de la catégorie de rationalité trouve son origine théorique dans l'objectivation du regard subjectif de sa sociologie : la "possibilité de réussite moyenne“" sur laquelle il s'appuie est une catégorie subjective, qui ne devient objective qu'à travers le concept de ratio ${ }^{13}$.

11 L'action rationnelle est elle-même un mode de comportement subjectif qui se distingue par le fait de rendre comparable les comportements sociaux, au-delà des motivations particulières des sujets. À l'intérieur de ce cadre, qui est donné par avance, le sociologue peut procéder à une interprétation objective. Adorno précise ce problème dans l'un de ses nombreux articles sociologiques : «La génération de Max Weber a rappelé à juste titre que tout idéal de compréhension était toujours partiel, dans la mesure où il éliminait ce qui contrarie l'identification de celui qui comprend, au sein de la société. ${ }^{14}$ Pour saisir les motivations qui se trouvent à la racine de résistances - en apparence irrationnelles au vu des incitations à s'adapter au modèle social existant - il convient donc de chercher à comprendre ce qui ne s'identifie pas à la société.

12 Il est vrai que la traduction tardive, partielle et hétéroclite des écrits et travaux sociologiques d'Adorno et alli dans l'espace francophone n'assure pas une base très large pour mener la discussion sociologique. En témoigne le titre d'un ouvrage collectif publié à Paris en 2002, qui situe l'Ecole de Francfort «entre sociologie et philosophie ${ }^{15}$, ce qui semble la placer dans un entre-deux indéterminé. Pareil titre reprend la définition des disciplines en France, marquée par une forte polarité entre la sociologie empirique d'un côté, et de l'autre la philosophie politique ou spéculative. Je défendrais plutôt l'idée d'une tension forte et productive entre deux pôles, qui sont entrés dans une interaction de type dialectique pour donner lieu à une recherche sociale sui generis.

En France, plusieurs ouvrages ont posé la question de l'actualité de cette Théorie critique d'inspiration francfortoise au cours des dix dernières années ${ }^{16}$. Les textes originaux centrés sur la recherche sociale ont été traduits de manière récente et partielle, comme par exemple les Etudes sur la personnalité autoritaire en 2007 ou encore une partie des interventions sociologiques d'Adorno, intitulés Société pour le public francophone, publié chez un autre éditeur français en $2011^{17}$. Malheureusement, ni la courte préface d'Honneth, ni le collecteur de direction ne signalent le rapport de ces textes aux œuvres complètes de la sociologie d'Adorno. Je saisis donc l'occasion qui m'est offerte ici pour préciser que l'ouvrage francophone Société reprend en partie le premier volume des écrits sociologiques d'Adorno, sans aborder le second volet, et sans mentionner un volume complémentaire constitué par les Etudes sur la personnalité autoritaire. Pareil patchwork éditorial ne favorise pas la réception cohérente de ces contributions en tant qu'aspects complémentaires d'une sociologie critique originale.

14 Je propose de concevoir la Théorie critique dans une relation nouvelle de la conceptualisation critique et de la recherche empirique, travail qui débute avec la 
déconstruction de la philosophie de l'histoire. Dans ses Trois études sur Hegel, Theodor W. Adorno comprend que le déploiement du concept philosophique à travers l'histoire occidentale tait le travail et les expériences particulières qui effectuent cette histoire. Rapidement, la dynamique philosophique s'affranchit ici de la contrainte sociale et devient spéculation, tout en dominant la scène, ce qui fait que le général prime sur le particulier et que la philosophie hégélienne (ou marxiste) imagine une fin de l'histoire, où le concept aurait dépassé les aspérités du réel. Adorno dit pourquoi ce dénouement parfait ne se produira jamais ; la raison en est que le concept intellectuel n'est jamais assez précis pour épouser la réalité historique, trop riche, ambivalente et contradictoire pour être contenue dans un mot ou dans une idée. Dès lors, les aspects et éléments négligés, écartés, s'affirment en dehors de la totalisation conceptuelle qui veut posséder le monde et la contredisent, dans un travail de négativité à l'encontre de l'ordre existant. Plus tard, on retrouve ces arguments dans La dialectique négative ${ }^{18}$. Parallèlement, Adorno s'érige contre une science qui prétend maitriser la réalité sociale de manière formelle et univoque, notamment à travers les statistiques ${ }^{19}$. Le voilà engagé sur le champ de la sociologie, pour mieux en contrer les effets positivistes, ce qui l'oblige en même temps à accepter le principe de la recherche empirique pour approcher les situations particulières. Ainsi, il tire les conséquences de sa conviction que la théorie abstraite écrase le particulier, tout en sachant que ce ne sont pas les observations éparses qui sauraient fonder une Théorie critique de la société (Horkheimer parle à ce propos d'observations isolantes).

À mon sens, la distanciation critique du monde et l'approche empirique de l'expérience particulière entrent ici dans une correspondance conflictuelle, mais créative. Pour résumer ce cheminement intellectuel, le concept ne saurait absorber le monde et l'expérience du particulier, alors que l'expérience sociale a besoin d'un imaginaire conceptuel critique, afin de ne pas succomber à l'apparent réalisme du fait accompli. Tel est aussi le cheminement qu'explorent Negt et Kluge dans les mille pages de leur ouvrage Geschichte und Eigensinn. L'expérience sociologique et la critique conceptuelle ne forment donc pas nécessairement deux logiques séparées, mais l'interaction créative que je crois déceler n'apparait que sur le plan global des travaux de la Théorie critique ou du moins de l'œuvre complète d'Adorno, et non pas dans un exposé programmatique qui serait inscrit dans un seul livre. A contrario, cette vue d'ensemble me semble avoir disparu du programme actuel de l'Institut francfortois, où la seule étude sociologique citée est la première enquête d'Habermas (Student und Politi $k^{20}$ ). L'orientation philosophique et les thèmes de recherche semblent donc y coexister sans se rencontrer.

Axel Honneth a publié un ouvrage collectif pour discuter La dialectique négative, qui témoigne de son désaccord avec l'approche adornienne. Honeth pense qu'Adorno a organisé un clivage fondamental entre la raison et la réalité, afin de mettre à l'abri sa perception du monde des fausses identifications que celui-ci ne cesse d'engendrer ${ }^{21}$. L'interaction que je décris entre la critique conceptuelle et l'expérience empirique disparaît dans cette lecture au bénéfice d'une construction logique. Selon Honneth, Adorno aurait d'abord posé les catégories philosophiques et critiques fondamentales, avant de chercher à atteindre une pensée qui s'approprie les contenus concrets (par exemple les questions sociologiques). Au-delà de la clarification de la construction théorique chez Adorno, l'assertion de Honneth évoque sans doute sa propre conception méthodologique et scientifique. Comme nous allons le voir, la perception des classiques de la sociologie européenne diverge en effet profondément chez Adorno et Honneth. 
18 D'un point de vue théorique, Honneth n'accepte pas la critique du positivisme qu'Adorno a affirmée ${ }^{22}$. L'auteur de Minima moralia ${ }^{23}$ a suggéré une critique fondamentale de l'emploi des statistiques en sciences sociales, sans pour autant rejeter l'outil en soi. Lors de la « querelle sur le positivisme », il constate que la méthode objectivante et objectiviste des statistiques est singulièrement appropriée pour décrire les tendances fétichistes du monde administré qui marquent les mentalités sociales ${ }^{24}$. Ces procédures techniques de la sociologie répondent donc à une réalité coiffée par la loi des moyennes et des grands nombres, autour de laquelle les comportements s'orientent. La méthode est juste, en ce qu'elle correspond à une société fausse. Les statistiques contribuent ainsi à une définition exacte de la société comme fait accompli, mais ne font en aucun cas apparaitre les potentialités subjectives de son dépassement.

19 C'est précisément cette orientation que conteste Honneth. Il rappelle que selon Adorno, l'objet sociologique ne peut être défini de manière correcte que si l'on prend en compte ses caractéristiques qualitatives qui apparaissent à travers « les expériences subjectives » que les acteurs éprouvent, expériences qui ne peuvent être résumées par un concept schématique («schematisierender Begriff » chez Honneth). Après avoir souligné le risque d'une influence de jugements subjectifs donc arbitraires sur le processus scientifique que Honneth définit comme neutralisation de la subjectivité - il reproche finalement une attitude potentiellement antidémocratique à Adorno, dont la position théorique reviendrait à ne retenir des personnes capables de jugements assez différenciés et sensibles dans la formulation d'un constat social ${ }^{25}$. Si je l'ai bien compris, Honneth somme Adorno de choisir entre la sélection d'une élite susceptible de formuler un idéal scientifique (à la manière de la République des savants) et la confusion intellectuelle provoquée par le concert d'opinions individuelles peu renseignées.

Honneth, qui se réfère de manière positive à la sociologie de Parsons, reproche à Adorno de verser dans une métaphysique transcendantale, utopiste qui se fonde sur la séparation de la raison (que j'associe à la critique) et de la réalité (d'ordre empirique). J'ai signalé plus haut comment Adorno invoque, en effet, la résistance et la nausée envers la société établie, au nom d'une vie bonne qui s'affirme avant tout en tant que concept critique. Si la médiation de l'expérience sensible et du concept critique n'est pas définitivement établie par l'auteur de la Dialectique négative, l'orientation honnethienne qui rétablit une division du travail académique plus classique entre sociologie et philosophie, comporte à mon sens un grand inconvénient. Cet inconvénient se manifeste avec force dans la notion honnethienne "d'expérience pré-scientifique», qui désigne des expériences non formalisées des acteurs (qu'Adorno avait désignées comme expériences non réglementées). En affirmant une frontière autant sémantique que formelle entre l'expérience des acteurs et la modalité par laquelle la science sociale cherche à l'évaluer, il tend à reproduire précisément l'aporie de la sociologie traditionnelle, qui ne représente que les expériences que son propre mode de formalisation arrive à identifier. En reléguant le champ de l'expérience sensible des acteurs vers un domaine de l'expérience "pré-scientifique» et en définissant une unité académique entre philosophie et sociologie (ou raison et réalité), Honneth peine à saisir les expériences particulières et non conformes aux normes qui interviennent dans la formation d'espaces publics oppositionnels. Ces espaces se constituent précisément et explicitement à travers le concept sociologique de résistance qui se trouve au cœur de l'intervention adornienne de 1968. Ce concept a partie liée avec une praxis de la résistance à l'encontre de l'adaptation 
aveugle au marché, à la gestion bureaucratique de l'Etat, à l'industrie du spectacle et aux représentations fétichistes que répètent les mass-medias.

21 Les pourfendeurs du langage de la résistance sont à court d'arguments depuis que celle-ci s'affirme partout sous des formes empiriques. Deux recours s'offrent à eux. Stipuler cyniquement que "l'humanité n'existe pas", à la manière de Sloterdijk qui cite explicitement le théoricien nazi Carl Schmitt ${ }^{26}$ et qui considère qu'il faut abandonner les surnuméraires à leur sort au profit des plus performants (l'actualité du concept de résistance rappelle ici la résistance à la barbarie moderne). Ou alors, à une échelle intellectuelle moindre, la tentative de confondre résistance et moralisme, dans l'affirmation que l'indignation d'Hessel ne produirait rien (à la manière du journaliste Zemmour). Si le petit traité de Stéphane Hessel, Indignez-vous!, a inspiré des mouvements démocratiques en Espagne et ailleurs en Europe, cela est peut-être dû au fait qu'il convoque le meilleur des pensées critiques, depuis la première page où il cite les Thèses sur l'histoire de Walter Benjamin ${ }^{27}$, jusqu'à la dernière page qui termine sur un Résister c'est créer - créer, c'est résister, ce leitmotiv de Gilles Deleuze, lui-même inspiré de Spinoza. Dans l'abécédaire de Deleuze de 1980, la lettre $\mathrm{R}$ marque la résistance ${ }^{28}$. Le seul fait d'énoncer ce concept est un acte de résistance créative, disait-il. J'écris ici que la résistance est un concept sociologique vivant.

\section{NOTES}

1. HESSEL S., Indignez-vous !, Indigène éditions, collection « Ceux qui marchent contre le vent ", Montpellier, 2010, $32 \mathrm{p}$.

2. COHN-BENDIT D., « Exkurs über die soziologische Theorie » in Linksradikalismus, ed. Rohwolt, 1968, pp. 35-38.

3. CHÂTELET G., Vivre et penser comme des porcs, Gallimard, 2000 : « La matière première consensuelle se transforme en une unanimité populiste des majorités silencieuses qui n'est jamais innocente. À ce populisme classique se greffe désormais un nouveau populisme yuppie - un technopopulisme - qui entend bien afficher sa post-modernité carnassière ».

4. ADORNO T. W., « Industriegesellschaft oder Spätkapitalismus ? » in Aufsätze, Suhrkamp, Francfort sur le Main, 1971, pp.149-166.

5. Voir FERRARESE E., «L'influence de la théorie des systèmes dans l'œuvre d'Habermas », Variations N. 4, 2003.

6. Voir NEUMANN A., Kritische Arbeitssoziologie, ed. Schmetterling, 2010, p.168.

7. ADORNO T. W., op.cit.

8. NEUMANN A., op.cit. ; Conscience de casse. La sociologie critique de l'Ecole de Francfort, ed. Burozoïques, Paris, 2010, voir theoriecritique.com.

9. BRAUNSTEIN D., Adornos Kritik der politischen Ökonomie, transcript Verlag, Bielefeld, 2011.

10. Voir notamment la vulgate marxiste exposée dans « Die Frankfurter Schule im Lichte des Marxismus », Marxistische Blätter, 1972. 
11. ADORNO T. W., « Marx und die Grundbegriffe der Soziologie » in : Backhaus, Dialektik der Wertform, ça ira, 1997.

12. HONNETH A., La société du mépris, La Découverte, 2007, p.158.

13. ADORNO T. W., « Einleitung zu Emile Durkheim » (préface) in : Durkheim, Soziologie und Philosophie, Suhrkamp, 1976, p.21.

14. ADORNO T. W., Soziologische Exkurse, Europäische Verlagsanstalt, 1953.

15. ABENSOUR M., MUHLMANN G. (dir.), L'Ecole de Francfort : la Théorie Critique entre philosophie et sociologie, Kimé, 2002.

16. Voir notamment RENAULT E., SINTOMER Y. (dir.), Où en est la Théorie critique ?, La Découverte, 2003 ; NEUMANN A., VINCENT J-M., Sciences sociales et engagement, Variations n. 4, Syllepse, 2003 ; SAGRADINI L., SPURK J. (coord.), La théorie critique, héritages hérétiques, Variations n. 7, Parangon, 2005 ; VASSORT P., Crises, Ecole de Francfort et théorie critique, ed. du Croquant, à paraître 2012.

17. ADORNO T. W. (trad. FRAPPAT H.), Études sur la personnalité autoritaire, Allia, 2007 (titre original : Studien zum autoritären Charakter, Suhrkamp Taschenbuch 1973) et ADORNO T. W. (trad. ARNOUX P., CHIRST J.), Société : Intégration, Désintégration - Ecrit sociologiques, Payot, 2011.

18. ADORNO T. W., Dialectique négative (1966), trad. Collège de philosophie, Payot, 1978

19. ADORNO T. W., " Soziologie und empirische Forschung », in Schriften 8, Suhrkamp, Francfort, 1997, p. 199.

20. Voir http://www.ifs.uni-frankfurt.de/institut/programm.htm

21. HONNETH A., MENKE C., Theodor W. Adorno : Negative Dialektik, Akademie Verlag, Berlin, 2006, pp.19.

22. ADORNO T. W., « Der Positivismusstreit in der deutschen Soziologie » in Aufsätze zur Gesellschaftstheorie, Suhrkamp, 1972, pp. 167.

23. ADORNO T. W. (trad. Kaufholz E. et Ladmiral J-R.), Minima Moralia, Payot, 2003.

24. DEMIROVIÇ A., Der non-konformistische Intellektuelle, Suhrkamp, 1999, p. 751.

25. HONNETH A., « Zum Begriff der Philosophie » in HONNETH A., MENKE C., op.cit., p.19. 26. Voir SLOTERDIJK P., « Co-immunité globale », Multitudes n. 45, 2011. Au sujet de sa proposition d'abolir l'impôt des riches et de supprimer les allocations sociales : http:// www.zeit.de/2010/49/Sloterdijk-Reichensteuer

27. BENJAMIN W., Écrits, Gallimard, 2003.

28. http://contemporaneitesdelart.fr/quelle-place-pour-lart-en-ce-xxieme-siecle/rcomme-resistances-extrait-de-labecedaire-de-gilles-deleuze/

\section{AUTEUR}

\section{ALEXANDER NEUMANN}

Directeur de publication de Variations. 